\title{
SOME FORMULAS OF L. CARLITZ \\ ON HERMITE POLYNOMIALS
}

\section{S.K. CHATTERJEA and S.M. EAQUB ALI}

Department of Mathematics

University of Calcutta

Calcutta - 700019

India

(Received May 31, 1991)

ABSTRACT. We have used the idea of 'quasi inner product' introduced by L. R.

Bragg in 1986 to consider generating series

$$
\sum_{n=0}^{\infty} \frac{H_{n}^{2}(x) H_{n}^{2}(y) t^{n}}{2^{2 n}(n !)^{2}}
$$

studied by L. Carlitz in 1963 . The pecularity of the series is that there is $(n !)^{2}$ in the denominator, which has a striking deviation from the usual generating series containing $n$ ! in the denominator. Our generating function for the said generating series is quite different from that of Carlitz, but somewhat analogous to generating integrals derived by G.N. Watson (Higher Transcendental function Vo1.III, P 271-272 for the case of Legendre, Gegenbauer and Jacobi polynomials.

KEY WORDS AND PHRASES. Hermite polynomials, Generating function, Quasi inner product.

1980 AMS SUBJECT CLASSIFICATION CODE 33A65

1. INTRODUCTION.

In [1], L. Carlitz has obtained the following formula involving Hermite polynomials :

$$
\begin{gathered}
\sum_{n=0}^{\infty} \frac{H_{n}^{2}(x) H_{n}^{2}(y) t^{n}}{2^{2 n}(n !)^{2}}=\sum_{r=0}^{\infty} \frac{1}{2^{2 r}(r !)^{2}} \frac{t^{r}}{(1+t)^{2 r+1}} \exp \left\{\frac{2\left(x^{2}+y^{2}\right) t}{1+t}\right\} \\
\cdot H_{2 r}\left(x \sqrt{\frac{1-t}{1+t}}\right) H_{2 r}\left(y \sqrt{\frac{1-t}{1+t}}\right),
\end{gathered}
$$

which is equivalent to

$$
\begin{aligned}
& \sum_{n=0}^{\infty} \frac{t^{n}}{2^{2 n}(n !)^{2}} H_{n}^{2}\left(x \sqrt{\frac{1+t}{1-t}}\right) H_{n}^{2}\left(y \sqrt{\frac{1+t}{1-t}}\right) \\
& =\sum_{r=0}^{\infty} \frac{H_{2 r}(x) H_{2 r}(y)}{2^{2 r}(r !)^{2}} \frac{t^{r}}{(1+t)^{2 r+1}} \exp \left\{\frac{2\left(x^{2}+y^{2}\right)}{1-t}\right\} .
\end{aligned}
$$

He has also pointed out that the polynomial

$$
F_{n}(x, y)=\sum_{r=0}^{n}(-1)^{r} \frac{H_{2 r}(x) H_{2 r}(y)}{2^{2 r}(r !)^{2}} L_{n-r}^{2 r}\left(2 x^{2}+2 y^{2}\right)
$$

has the generating function 


$$
\sum_{n=0}^{\infty} F_{n}(x, y) t^{n}=\sum_{r=0}^{\infty} \frac{(-1)^{r} H_{2 r}(x) H_{2 r}(y) t^{r}}{2^{2 r}(r !)^{2}(1-t)^{2 r+1}} \exp \left\{\frac{-2 t\left(x^{2}+y^{2}\right)}{1-t}\right\},
$$

which suggests the problem of finding a closed expression for the sum

$$
i_{r=0}^{\infty} \frac{(-1)^{r} n_{2 r}(x) 4_{2 r}(y)}{2^{2 r}(r !)^{2}} z^{r} \text {. }
$$

The object of the present paper is first to prove the formula

$$
\begin{aligned}
& \sum_{n=0}^{\infty} \frac{H_{n}^{2}(x) \eta_{n}^{2}(y) t^{n}}{2^{2 n}(n !)^{2}} \\
& =\frac{e^{x^{2}+y^{2}}}{2 \pi} \int_{0}^{2 \pi}\left(1-2 t \cos 2 \theta+t^{2}\right)^{-\frac{1}{2}} \exp \left\{\frac{(t-1)\left(x^{2}+y^{2}\right)}{1+2 \sqrt{ } t \cos \theta+t}\right\} \cos \left\{\frac{2 \sqrt{ } t\left(x^{2}-y^{2}\right) \sin \theta}{1+2 \sqrt{t} \cos \theta+t}\right\} d \theta,(1.5)
\end{aligned}
$$

which may be compared with the formula (1.1) due to Carlitz.

Indeed, on comparing (1.1) and (1.5) we obtain

$$
\sum_{r=0}^{\infty} \frac{1}{2^{2 r}(r !)^{2}} \frac{t^{r}}{(1+t)^{2 r+1}} H_{2 r}\left(x \sqrt{\frac{1-t}{1+t}}\right) H_{2 r}\left(y \sqrt{\frac{1-t}{1+t}}\right)=\exp \left\{\frac{1-t}{1+t}\left(x^{2}+y^{2}\right)\right\} I \text {, }
$$

where

$$
I=\frac{1}{2 \pi} \int_{0}^{2 \pi}\left(1-2 t \cos 2 \theta+t^{2}\right)^{-\frac{1}{2}} \exp \left\{\frac{(t-1)\left(x^{2}+y^{2}\right)}{1+2 \sqrt{t} \cos \theta+t}\right\} \cos \left\{\frac{2 \sqrt{ } t\left(x^{2}-y^{2}\right) \sin \theta}{1+2 \sqrt{t} \cos \theta+t}\right\} d \theta .
$$

The following particular cases of our result (1.5) seem to be interesting,

$$
\begin{aligned}
& \sum_{n=0}^{\infty} \frac{H_{n}^{4}(x) t^{n}}{2^{2 n}(n !)^{2}}=\frac{e^{2 x^{2}}}{2 \pi} \int_{0}^{2 \pi}\left(1-2 t \cos 2 \theta+t^{2}\right)^{-\frac{1}{2}} \exp \left\{\frac{2 x^{2}(t-1)}{1+2 \sqrt{t} \cos \theta+t}\right\} d \theta \\
& \sum_{m=0}^{\infty} \frac{(2 m !)^{2}}{(m !)^{4}}(t / 4)^{2 m}=\frac{1}{2 \pi} \int_{0}^{2 \pi}\left(1-2 t \cos 2 \theta+t^{2}\right)^{-\frac{1}{2}} d \theta .
\end{aligned}
$$

Similarly from our result (1.6) the following particular cases are noteworthy :

$$
\begin{aligned}
& \sum_{r=0}^{\infty} \frac{1}{2^{2 r}(r !)^{2}} \frac{t^{r}}{(1+t)^{2 r+1}} H_{2 r}^{2}(x) \\
& =\frac{e^{2 x^{2}}}{2 \pi} \int_{0}^{2 \pi}\left(1-2 t \cos 2 \theta+t^{2}\right)^{-\frac{1}{2}} \exp \left\{\frac{-2(1+t) x^{2}}{1+2 \sqrt{t} \cos \theta+t}\right\} d \theta . \\
& \sum_{r=0}^{\infty} \frac{(2 r !)^{2} t^{r}}{2^{2 r}(r !)^{4}(1+t)^{2 r+1}}=\frac{1}{2 \pi} \int_{0}^{2 \pi}\left(1-2 t \cos 2 \theta+t^{2}\right)^{-\frac{1}{2}} d \theta .
\end{aligned}
$$

2. PROOF OF OUR RESULT (1.5).

In proving the result (1.5) we recall the method of 'quasi inner product', introduced by L.R. Bragg [2], where the name 'quasi inner product' is not appropriate, on account of the fact that inner product has a different meaning, however one may call it simply the product or multiplication like Hadamard's multiplication.

We know that

$$
\sum_{n=0}^{\infty} H_{n}^{2}(x) \frac{t^{n}}{n !}=\left(1-4 t^{2}\right)^{-\frac{1}{2}} \exp \left(\frac{4 x^{2} t}{1+2 t}\right) \equiv f(x, t)
$$


Similarly we write

$$
\sum_{n=0}^{\infty} \frac{Y_{n}^{2}(y) t^{n}}{n !}=\left(1-4 t^{2}\right)^{-1} \exp \left(\frac{4 y^{2} t}{1+2 t}\right) \equiv f(y, t)
$$

Then by Pragg's method we obtain

$$
\begin{aligned}
& f(x, t) \circ f(y, t)=\sum_{n=0}^{\infty} \cdot \frac{y_{n}^{2}(x) H_{n}^{2}(y) t^{2 n}}{(n !)^{2}} \\
& =\frac{1}{2 \pi} \int_{0}^{2 \pi}\left(1-4 t^{2} e^{2 i \theta}\right)^{-\frac{1}{2}}\left(1-4 t^{2} e^{-2 i \theta}\right)^{-\frac{1}{2}} \exp \left\{\frac{4 x^{2} t e^{i \theta}}{1+2 t e^{i \theta}}+\frac{4 y^{2} t e^{-i \theta}}{1+2 t e^{-i \theta}}\right\} d \theta \\
& =\frac{1}{2 \pi} \int_{0}^{2 \pi}\left(1-3 t^{2} \cos 2 \theta+15 t^{4}\right)^{-\frac{1}{2}} \exp \left\{4 t \frac{\left(x^{2}+y^{2}\right) \cos \theta+2 t\left(x^{2}+y^{2}\right)}{1+4 t \cos \theta+4 t^{2}}\right\} \\
& \quad \cdot \cos \left\{\frac{4 t\left(x^{2}-y^{2}\right) \sin \theta}{1+4 t \cos \theta+4 t^{2}}\right\} d \theta .
\end{aligned}
$$

In other words

$$
\begin{aligned}
\sum_{n=0}^{\infty} \frac{H_{n}^{2}(x) H_{n}^{2}(y) t^{n}}{2^{2 n}(n !)^{2}}=\frac{e^{x^{2}+y^{2}}}{2 \pi} \int_{0}^{2 \pi}\left(1-2 t \cos 2 \theta+t^{2}\right)^{-\frac{1}{i}} \exp \left\{\frac{(t-1)\left(x^{2}+y^{2}\right)}{1+2 \sqrt{t} \cos \theta+t}\right\} \\
\quad \cdot \cos \left\{\frac{2 \sqrt{t}\left(x^{2}-y^{2}\right) \sin \theta}{1+2 \sqrt{t} \cos \theta+t}\right\} d \theta
\end{aligned}
$$

which is (1.5).

\section{PARTICULAR CASES.}

Using $y=x$ in (1.5) we get

$$
\sum_{n=0}^{\infty} \frac{y_{n}^{4}(x) t^{n}}{2^{2 n}(n !)^{2}}=\frac{e^{2 x^{2}}}{2 \pi} \int_{0}^{2 \pi}\left(1-2 t \cos 2 \theta+t^{2}\right)^{-\frac{1}{2}} \exp \left\{\frac{2(t-1) x^{2}}{1+2 \sqrt{t} \cos \theta+t}\right\} d \theta \text {, }
$$

which is (1.8).

Again putting $x=0$ in (3.1) we get

$$
\sum_{m=0}^{\infty} \frac{(2 m !)^{2}}{(m !)^{4}}\left(\frac{t}{4}\right)^{2 m}=\frac{1}{2 \pi} \int_{0}^{2 \pi}\left(1-2 t \cos 2 \theta+t^{2}\right)^{-\frac{1}{2}} d \theta,
$$

which is (1.9).

It may be noted that the integrnd is connected with the generating function of the Legendre polynomials.

Indeed, using the well-known generating function of the Legendre polynomials and term-by-term integration we obtain

$$
\frac{1}{2 \pi} \int_{0}^{2 \pi}\left(1-2 t \cos 2 \theta+t^{2}\right)^{-\frac{1}{2}} d \theta=\frac{1}{2 \pi} \sum_{n=0}^{\infty} t^{n}\left(\int_{0}^{2 \pi} P_{n}(\cos 2 \theta) d \theta\right) .
$$

Now by the known result

$$
\begin{aligned}
\frac{1}{2 \pi} \int_{0}^{2 \pi} P_{n}(\cos \theta) d \theta & =0 \text { when } n \text { is odd } \\
& =\frac{(2 m !)^{2}}{(m !)^{4}} 4^{-2 m}, \text { when } n=2 m .
\end{aligned}
$$

We can rediscover $(3.2)$. 
The derivation of the particular case (1.10) and (1.11) are similar in nature.

Furthermore, it may be of much interest to compare (1.9) and (1.11) as the left members of two results are apparently different in nature. We now like to examine the left members of (1.9) and (1.11). To do this we first observe that the left member of $(1.11)$ is

$$
\sum_{m=0}^{\infty} \frac{(2 m !)^{2} t^{m}}{2^{2 m}(m !)^{4}(1+t)^{2 m+1}}=\sum_{m=0}^{\infty} t^{m} \sum_{r=0}^{m} \frac{(-1)^{r}(2 m-2 r) !(2 m-r) !}{r ! 2^{2(m-r)}((m-r) !)^{4}},
$$

which, on comparison with the series in the left member of (1.9), yields the novel identity

$$
\begin{aligned}
\sum_{r=0}^{m} \frac{(-1)^{r}(2 m-2 r) !(2 m-r) !}{r ! 2^{2(m-r)}((m-r) !)^{4}} & =0 \text { for odd } n . \\
& =\frac{(m !)^{2}}{((m / 2) !)^{4}} \frac{1}{4^{m}} \text { for even } m .
\end{aligned}
$$

\section{REFERENCES}

1. Bragg, L.R.

2. Carlitz, L.
: Quasi inner products of analytical functions with application to special functions, SIAM J. MATH. ANAL. Vol.17 (1986), 220-230.

: Some formulas of E. Feldheim, Acta Math. Acad. Sci. Hungar 14 (1963), P 21-29. 


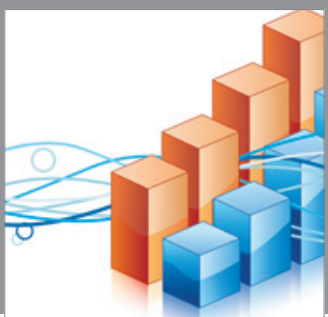

Advances in

Operations Research

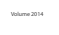

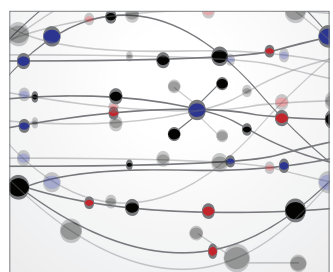

\section{The Scientific} World Journal
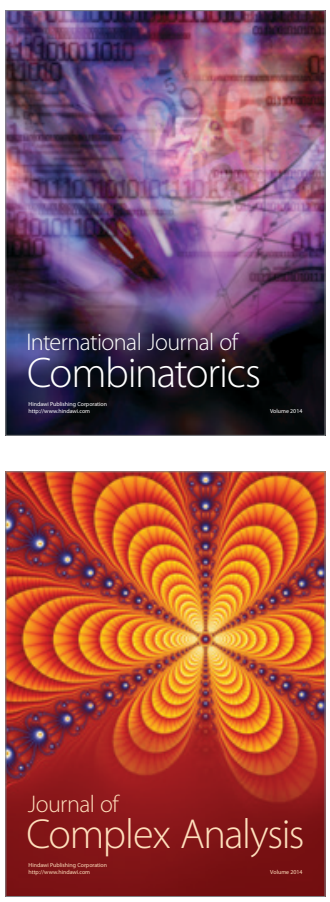

International Journal of

Mathematics and

Mathematical

Sciences
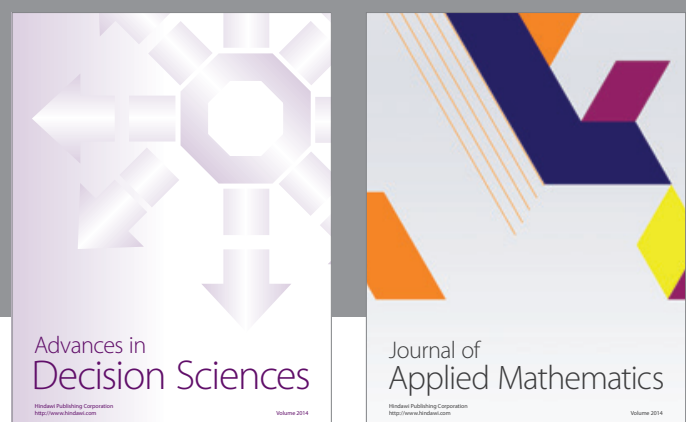

Journal of

Applied Mathematics
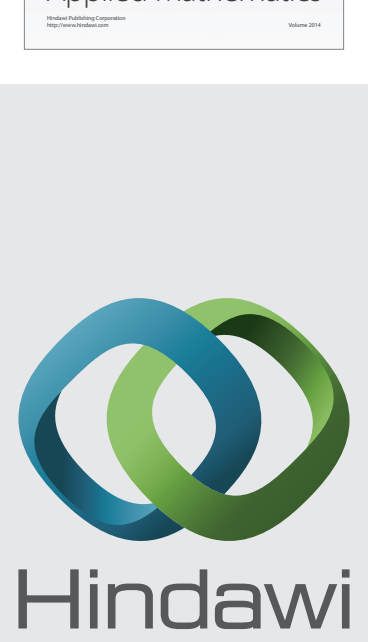

Submit your manuscripts at http://www.hindawi.com
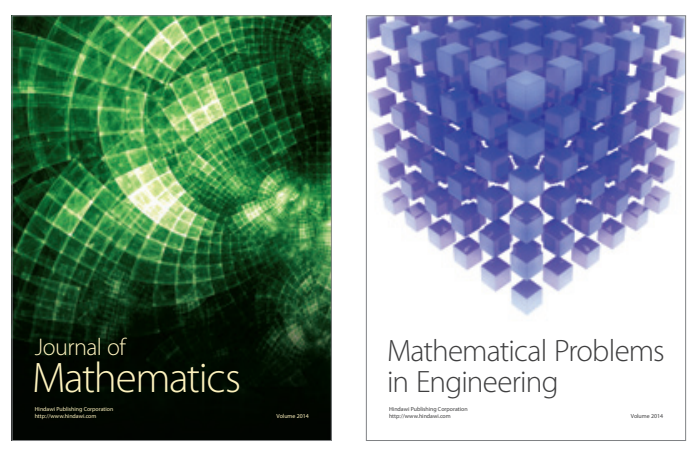

Mathematical Problems in Engineering
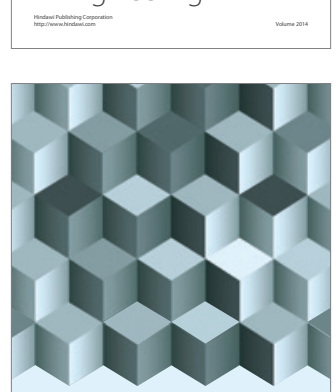

Journal of

Function Spaces
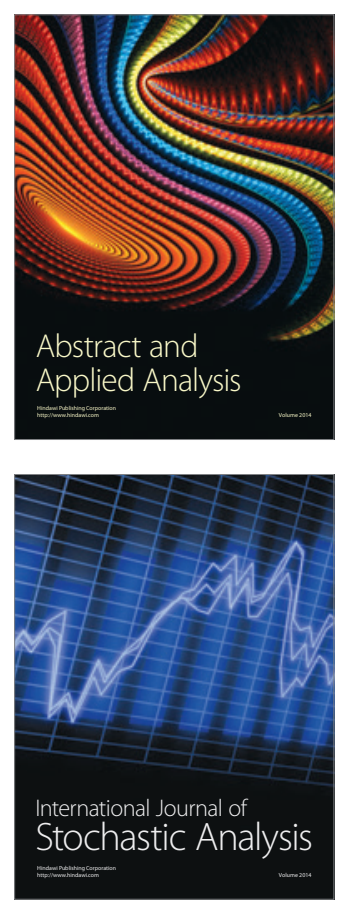

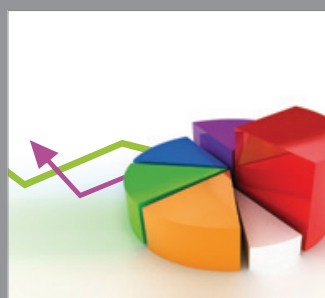

ournal of

Probability and Statistics

Promensencen
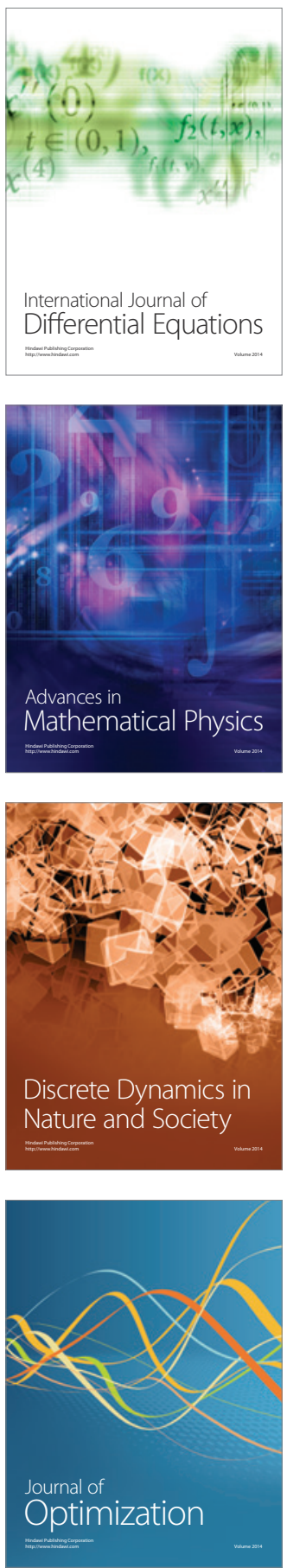